\title{
On Kalman Filtering with Fading Wireless Channels Governed by Power Control *
}

\author{
Daniel E. Quevedo* Anders Ahlén ${ }^{* *}$ Alex S. Leong ${ }^{* * *}$ \\ Subhrakanti Dey ${ }^{* * *}$ \\ * School of Electrical Engineering 85 Computer Science, The University \\ of Newcastle, NSW 2308, Australia (e-mail: dquevedo@ieee.org). \\ ** Department of Engineering Sciences, Signals and Systems, Uppsala \\ University, P. O. Box 534, SE-751 21 UPPSALA, Sweden (e-mail: \\ anders.ahlen@signal.uu.se) \\ *** Department of Electrical and Electronic Engineering, The \\ University of Melbourne, Parkville, VIC 3052 (e-mails: \\ asleong@unimelb.edu.au,sdey@unimelb.edu.au)
}

\begin{abstract}
We study stochastic stability for Kalman filtering over fading wireless channels where variable channel gains are counteracted by the use of power control to alleviate the effects of packet drops. The Kalman filter and the controller are located at a single gateway which acquires data from the wireless sensors. We establish sufficient conditions which ensure that the Kalman filter covariance matrix is exponentially bounded in norm. The conditions obtained are then used to formulate stabilizing optimal power allocation laws which minimize the total sensor power budget. In deriving the optimal power allocation laws, both statistical channel information and full channel information are considered. The effect of system instability on the power budget is also investigated for both these cases.
\end{abstract}

Keywords: State estimation, fading channels, packet dropouts, stochastic stability, power control.

\section{INTRODUCTION}

The interest in estimation and control over communication channels subject to packet loss has increased tremendously in recent years; see, e.g., Hespanha et al. (2007); Nair et al. (2007); Schenato et al. (2007). First, the rapid evolution of Wireless Sensor Networks, see e.g., Ilyas et al. (2004); Shen et al. (2007); Havinga et al. (2004); Gharavi and Kumar (2003) has made wireless sensors cheap and reliable enough to be brought into commercial use. Second, wireless sensors offer several advantages for industrial control systems, such as, flexibility, low cost, and fast deployment. Third, with wireless sensors electrical contact problems are no longer an issue. Furthermore, wireless sensors and actuators can be placed where wires cannot go, or where power sockets are unavailable.

Wireless communication channels on the other hand are frequently subject to fading and interference, which may lead to packet errors. A key aspect is that fading channels can, at least partially, be compensated for by adjusting the transmission power levels. The use of suitable power control algorithms, thus, becomes an important issue for reliably acquiring measurements from wireless sensors.

Depending on the situation at hand, wireless channels can either be static (the environment is static and receiver and transmitter are not moving), or it can be time varying. The time variations can either be slow, which is frequently

^ This research was supported under Australian Research Council's Discovery Projects funding scheme (project number DP0988601). the case when large objects obstruct the propagation channel, or they can be rapid, which is often the case when transmitters or receivers are mounted on moving objects, or when objects are moving between them. The degree of variability will also depend on the wireless systems radio interface and its bandwidth, see, e.g., Goldsmith (2005).

Apart from fading channels, which may cause packet drops, another important issue, which arises when wireless sensors are used, is that in the absence of power sockets, there is a need for energy conservation. Even though energy harvesting is frequently considered a remedy for battery powered sensor nodes, today's technology does not suffice in many industrial applications. Thus, saving energy is of utmost importance to avoid unnecessary maintenance and replacement of batteries. We conclude that packet loss will unavoidably occur at the receivers. Depending on how severe the fading is and how much power is available to counteract it, packets will be dropped more or less frequently.

Recently, energy issues have attracted significant attention in the wireless sensor networks literature; see, e.g., Björnemo (2009); Wen et al. (2008); Xiao et al. (2008); Björnemo et al. (2007); Johansson et al. (2007). For example, in Xiao et al. (2008) convex optimization is used to derive optimal power scheduling for a time-invariant decentralized case. Power control will be a highly useful tool to counteract time variations in wireless links. Since energy is severely limited in most wireless sensor applications, power control design involves trading energy consumption 


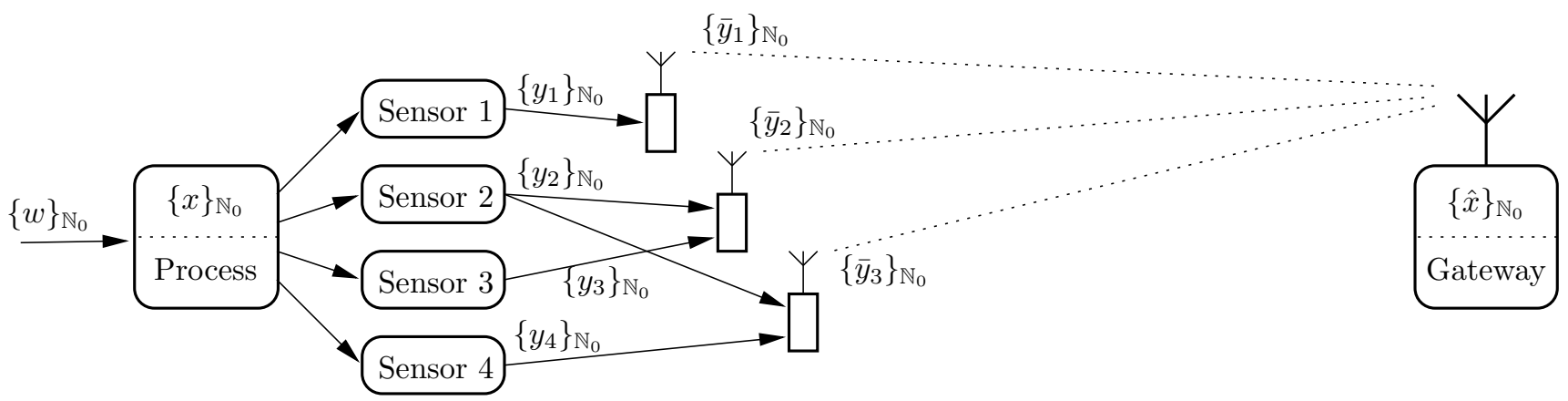

Fig. 1. State estimation with $M=4$ wireless sensors and $L=3$ wireless channels. The radio at the top is transmitting values from Sensor 1 only, whereas the middle and bottom radios combine into one packet the values from Sensor 2 and 3, and Sensor 2 and 4, respectively.

for accuracy. This was considered in the context of state estimation with power controlled sensor data transmitted over fading channels, e.g., in Quevedo et al. (2010).

In the present work we study state estimation with a time-varying Kalman filter performing optimal state estimation at a gateway. Sensor data are transmitted over fading channels generating random packet loss. The packet loss probabilities depend, in a nonlinear fashion, upon the time-varying channel gains and power levels used by the sensors. Here we focus on centralized control of the transmission power levels to be used by each sensor. We establish sufficient conditions which ensure that the Kalman filter covariance matrix is exponentially bounded in norm. The conditions obtained are then used to formulate stabilizing optimal power allocation laws which minimize the total power used by the sensors.

Before proceeding, we note that stability of time-varying Kalman filters in the face of random packet loss has recently been studied extensively. Representative works include Sinopoli et al. (2004); Xie and Xie (2008); Schenato (2008); Kluge et al. (2010); Gupta et al. (2009); Shi et al. (2010); Dey et al. (2009). However, to the best of the authors' knowledge, such kind of stability analysis has not been performed previously for the case of state estimation with power controlled digital wireless links.

The remainder of this paper is organized as follows: In Section 2, we present the process model and the structure of the wireless links used. Section 3 describes the state estimator with packet drops and power control, and presents a sufficient condition for stochastic stability of the estimator. In Section 4, the stability result is used to perform optimum power allocation. An example is included to compare optimum static and dynamic power allocations. Finally, in Section 5 conclusions are drawn.

Notation: We write $\mathbb{N}_{0}$ for $\{0,1,2,3, \ldots\},\{\nu\}_{\mathbb{N}_{0}}$ for $\{\nu(0), \nu(1), \ldots\}$, and $\{\nu\}_{0}^{k}$ for $\{\nu(0), \nu(1), \ldots, \nu(k)\}$. A random variable $\nu(k)$, which is Gaussian with mean value $m$ and covariance matrix $\Gamma$ is denoted $\nu(k) \sim \mathcal{N}(m, \Gamma)$. Absolute value is denoted by $|\cdot|$. Furthermore, min eigs $A$ and max eigs $A$ denote the minimum and maximum of the set of eigenvalues of a matrix $A$, respectively. The trace of a matrix $A$ is denoted by $\operatorname{tr} A$, and the norm of the same matrix by $\|A\| \triangleq \sqrt{\max \operatorname{eigs}\left(A^{T} A\right)}$. If a matrix $A$ is positive definite (semi definite), then we write $A \succ 0$
( $A \succeq 0)$. To denote the conditional probability of an event $\Omega$ given $\Delta$, we write $\mathcal{P}\{\Omega \mid \Delta\}$; the unconditional probability is denoted $\mathcal{P}\{\Omega\}$. The probability density (mass) function of a (discrete) random variable $\nu$ is denoted as $p(\nu)$, its expected value is denoted via $\mathbb{E}\{\nu\}$, whereas for the conditional expectation given $\Delta$, we write $\mathbb{E}\{\nu \mid \Delta\}$. We use the same notation for random variables and their realizations. What is meant will depend on the context.

\section{ESTIMATION ARCHITECTURE}

Consider an uncontrolled LTI $n$-dimensional system:

$$
x(k+1)=A x(k)+w(k), \quad k \in \mathbb{N}_{0},
$$

where the initial system state $x(0) \sim \mathcal{N}\left(x_{0}, P_{0}\right)$. The driving noise process $\{w\}_{\mathbb{N}_{0}}$ is independent and identically distributed (i.i.d.), where each $w(k) \sim \mathcal{N}(0, Q), Q \succ 0$. To remotely estimate the system state sequence $\{x\}_{\mathbb{N}_{0}}, M$ wireless sensors are used. Each sensor provides a scalar noisy measurement sequence $\left\{y_{m}\right\}_{\mathbb{N}_{0}}$, where

$$
y_{m}(k)=C_{m} x(k)+v_{m}(k), \quad m \in\{1,2, \ldots, M\} .
$$

In (2), $\left\{v_{m}\right\}_{\mathbb{N}_{0}}$ is an i.i.d. scalar measurement noise process with each $v_{m}(k) \sim \mathcal{N}\left(0, \sigma_{m}^{2}\right)$, with $\sigma_{m}^{2}>0$

The $M$ measurements in (2) are to be transmitted to a single gateway via $L$ wireless links. Upon receipt, the signals are used to remotely estimate the state of the system (1). The sensor measurements can be transmitted to the gateway in several ways. To be more specific, the collected values from the $M$ sensors, namely, $\mathbb{Y}(k) \triangleq$ $\left\{y_{1}(k), y_{2}(k), \ldots, y_{M}(k)\right\}$ are combined into $L$ packets $\left\{\bar{y}_{1}(k), \bar{y}_{2}(k), \ldots, \bar{y}_{L}(k)\right\}$ where each packet contains measurements of one or more sensors, i.e., $\bar{y}_{\ell}(k) \subseteq \mathbb{Y}(k)$. The values transmitted over each wireless link, thus amount to a vector valued measurement sequence, say $\left\{\bar{y}_{\ell}\right\}_{\mathbb{N}_{0}}$, where

$$
\bar{y}_{\ell}(k)=\bar{C}_{\ell} x(k)+\bar{v}_{\ell}(k), \quad \ell \in\{1,2, \ldots, L\} .
$$

The associated observation noise $\left\{\bar{v}_{\ell}\right\}_{\mathbb{N}_{0}}$ is an i.i.d. process with $\bar{v}_{\ell}(k) \sim \mathcal{N}\left(0, R_{\ell}\right)$, where $R_{\ell}$ is a diagonal matrix containing the corresponding individual covariances $\sigma_{m}^{2}$. The rows of the observation matrices $\bar{C}_{\ell}$ are the corresponding row vectors $C_{m}$, see (2).

Example 1. Fig. 1 depicts a particular estimation architecture with $M=4$ sensors and $L=3$ links and where

$$
\begin{aligned}
& \bar{y}_{1}(k)=y_{1}(k), \quad \bar{y}_{2}(k)=\left[\begin{array}{l}
y_{2}(k) \\
y_{3}(k)
\end{array}\right], \quad \bar{y}_{3}(k)=\left[\begin{array}{l}
y_{2}(k) \\
y_{4}(k)
\end{array}\right] \\
& \bar{C}_{1}(k)=C_{1}(k), \quad \bar{C}_{2}(k)=\left[\begin{array}{l}
C_{2}(k) \\
C_{3}(k)
\end{array}\right], \quad \bar{C}_{3}(k)=\left[\begin{array}{l}
C_{2}(k) \\
C_{4}(k)
\end{array}\right] .
\end{aligned}
$$




\section{STATE ESTIMATION WITH WIRELESS SENSORS}

In this section, we formulate the state estimation architecture in the face of packet drop-outs caused by fading channels. We then present sufficient conditions for stochastic boundedness of the covariance of the estimation error.

\subsection{Effects of Fading Channels}

Since the $L$ links used to convey measurements between sensors and the gateway are wireless, transmission errors are likely to occur. Thus, faulty packets will frequently be received. Such packets are discarded when estimating the system state sequence $\{x\}_{\mathbb{N}_{0}}$. We model transmission effects on a wireless link basis by introducing the $L$ binary stochastic arrival processes $\left\{\gamma_{\ell}\right\}_{\mathbb{N}_{0}}, \ell \in\{1, \ldots, L\}$, where:

$\gamma_{\ell}(k)= \begin{cases}1 & \text { if } \bar{y}_{\ell}(k) \text { arrives error-free at time } k, \\ 0 & \text { if } \bar{y}_{\ell}(k) \text { does not arrive error-free at time } k .\end{cases}$

For future reference, we also define the overall arrival process of all $L$ links, say $\{\gamma\}_{\mathbb{N}_{0}}$, via:

$$
\gamma(k) \triangleq\left[\gamma_{1}(k) \gamma_{2}(k) \ldots \gamma_{L}(k)\right], \quad k \in \mathbb{N}_{0} .
$$

The success probabilities of the processes $\left\{\gamma_{\ell}\right\}_{\mathbb{N}_{0}}$ are, in general, time-varying and depend upon the propagation environment and on the transmission power used by the sensor radio power amplifiers. Indeed, by adopting a blockfading model, the conditional transmission success probabilities satisfy:

$$
\mathcal{P}\left\{\gamma_{\ell}(k)=1 \mid h_{\ell}(k), u_{\ell}(k)\right\}=f_{\ell}\left(h_{\ell}(k) u_{\ell}(k)\right),
$$

where $u_{\ell}(k)$ denotes the power used by the $\ell$-th radio power amplifier, whereas $h_{\ell}(k)$ is the corresponding channel power gain. The functions $f_{\ell}(\cdot):[0, \infty) \rightarrow[0,1]$ are monotonically increasing and differentiable, and depend upon the modulation scheme employed; see, e.g., Proakis (1995). In Section 4.2, we will investigate a specific case.

\subsection{State Estimation with Packet Drop-outs}

We will assume that the packets transmitted from the sensors to the gateway incorporate error detection coding; see, e.g., Proakis (1995). Hence, the gateway knows, whether received packets, containing collections of sensor measurements, are correct or not. Thus, at any time $k$, past and present realizations of the process $\{\gamma\}_{\mathbb{N}_{0}}$ are available at the gateway. For state estimation purposes, the system amounts to sampling (1), (3) with the stochastic output matrix:

$$
C(k) \triangleq\left[\begin{array}{c}
\gamma_{1}(k) \bar{C}_{1} \\
\gamma_{2}(k) \bar{C}_{2} \\
\vdots \\
\gamma_{L}(k) \bar{C}_{L}
\end{array}\right]
$$

The conditional probability distribution of $x(k)$, given $\{\gamma\}_{0}^{k}$ and correctly received packets with sensor measurements up to time $k$, i.e., $\{\bar{y}\}_{0}^{k}$, is Gaussian.

Consequently, the conditional means of $x(k)$ and the (prior) covariance of the state estimation error, i.e.,

$\hat{x}(k) \triangleq \mathbb{E}\left\{x(k) \mid\{y\}_{0}^{k},\{\gamma\}_{0}^{k}\right\}$

$\check{x}(k) \triangleq \mathbb{E}\left\{x(k) \mid\{y\}_{0}^{k-1},\{\gamma\}_{0}^{k-1}\right\}$

$P(k) \triangleq \mathbb{E}\left\{(x(k)-\check{x}(k))(x(k)-\check{x}(k))^{T} \mid\{y\}_{0}^{k-1},\{\gamma\}_{0}^{k-1}\right\}$, satisfy the Kalman filter recursions (see, e.g., Anderson and Moore (1979)):

$$
\begin{aligned}
& \check{x}(k+1)=A \hat{x}(k) \\
& \hat{x}(k+1)=A \hat{x}(k)+K(k+1)(y(k+1)-C(k+1) A \hat{x}(k)) \\
& P(k+1)=A P(k) A^{T}+Q-A K(k) C(k) P(k) A^{T}
\end{aligned}
$$

with initial values $P(0)=P_{0}$ and $\hat{x}(-1)=x_{0}$, and where

$$
\begin{aligned}
K(k) & \triangleq P(k) C(k)^{T}\left(C(k) P(k) C(k)^{T}+R\right)^{-1} \\
R & \triangleq \operatorname{diag}\left(R_{1}, R_{2}, \ldots, R_{L}\right) \succ 0 .
\end{aligned}
$$

Note that, since $\{C\}_{\mathbb{N}_{0}}$ is a random process, so is $\{P\}_{\mathbb{N}_{0}}$. An important question arises as to whether the above state estimator is stable, i.e., whether $\{P\}_{\mathbb{N}_{0}}$ is bounded. This issue will be studied below.

\subsection{Stochastic Stability}

Due to fading channels, the sequence of error covariances of the state estimator (7) becomes a stochastic process. Properties of $\{P\}_{\mathbb{N}_{0}}$ are closely related to those of $\{C\}_{\mathbb{N}_{0}}$ defined in (6). Since the arrival processes $\left\{\gamma_{\ell}\right\}_{\mathbb{N}_{0}}$ are binary, the process $\{C\}_{\mathbb{N}_{0}}$ is discrete, where each $C(k)$ takes one of $2^{L}$ possible values. The distribution of $C(k)$ depends upon the current channel gains $h_{\ell}(k)$ and the control law adopted; see (5) and (6). In general, $P(k)$ will not converge to a fixed value. Theorem 1, stated below, characterizes stability properties of $\{P\}_{\mathbb{N}_{0}}$. To state our result, we introduce the random process $\{s\}_{\mathbb{N}_{0}}$, where:

$$
s(k)= \begin{cases}1 & \text { if } C(k) \text { has full column-rank }, \\ 0 & \text { otherwise. }\end{cases}
$$

Theorem 1. Suppose that the process $\{\gamma\}_{\mathbb{N}_{0}}$ defined in (4) is white ${ }^{1}$ and that there exists $\rho \in[0,1)$, such that:

$$
\mathcal{P}\{s(k)=1\} \geq 1-\frac{\rho}{\|A\|^{2}}, \quad \forall k \in \mathbb{N} .
$$

Then, $\{P\}_{\mathbb{N}_{0}}$ is exponentially bounded in norm, i.e., there exist finite constants $\alpha$ and $\beta$, such that:

$$
\mathbb{E}\{\|P(k)\|\} \leq \alpha \rho^{k}+\beta, \quad \forall k \in \mathbb{N}_{0} .
$$

\section{Proof. See Appendix A.}

The above theorem establishes sufficient conditions for stochastic stability of the covariance matrix $\{P\}_{\mathbb{N}_{0}}$, i.e., of the time-varying Kalman filter used for state estimation over wireless fading channels with power control. It is worth noting that the condition on $\mathcal{P}\{s(k)=1\}$, or bounds thereof, can at times be calculated by conditioning upon, for example, channel state distribution, channel gain distribution, power control policy, etc. In the following section, we will take a closer look at how Theorem 1 can be used in various power control settings.

Remark 2. Situations with fixed success probabilities

$$
\mathcal{P}\left\{\gamma_{\ell}(k)=1\right\}=\lambda_{\ell} \in[0,1], \quad \forall k \in \mathbb{N}_{0}
$$

have been extensively studied previously in the literature; see, e.g, Schenato et al. (2007); Shi et al. (2010); Sinopoli et al. (2004); Gupta et al. (2009); Rohr et al. (2010). For example, for the one sensor-link case, Sinopoli et al. (2004)

1 That is, the random variables $\gamma(\ell), \ell \in \mathbb{N}_{0}$ are independent in time (but not necessarily identically distributed). 
established that there exists a critical transmission success probability, which determines stability of the estimator. Our result in Theorem 1 complements those works by considering transmission over multiple wireless channels with time-varying success probabilities, see (5).

\section{CONTROL OF TRANSMISSION POWERS}

It follows from the discussion so far that one can improve transmission reliability and, thus, state estimation accuracy for a given wireless propagation environment, by simply increasing the power used by the transmitters. However, in wireless sensor networks, it is of fundamental importance to save energy. Furthermore, the transmission power available to the individual sensors will also be constrained in magnitude. Thus, power control design involves a trade-off between transmission error probabilities (and, thus, state estimation accuracy) and energy consumption, see also Quevedo et al. (2010). Whilst it is indeed important to balance state estimation accuracy against the power used, it is essential to allocate enough power to the sensor nodes so that exponentially boundedness in norm of the state estimation covariance matrix $P(k)$ can be guaranteed. We will consider this issue next.

\subsection{Stabilizing Power Allocation Strategies}

The stability result provided in Theorem 1 can be applied to settings of various degrees of complexity. In the remainder of this work, we will focus on an estimation architecture where the number of sensors is equal to the state dimension. The vectors $\left\{C_{1}, \ldots, C_{M}\right\}$ are linearly independent and each sensor transmits over a dedicated channel. In this case, we have $n=M=L$ and the output matrix becomes

$$
C(k)=\left[\begin{array}{c}
\gamma_{1}(k) C_{1} \\
\gamma_{2}(k) C_{2} \\
\vdots \\
\gamma_{M}(k) C_{M}
\end{array}\right] \in \mathbb{R}^{M \times M} .
$$

Thus, $C(k)$ has full rank, if and only if $\gamma_{\ell}(k)=1, \forall \ell \in$ $\{1,2, \ldots, M\}$. Accordingly, $\{s\}_{\mathbb{N}_{0}}$ becomes:

$$
s(k)=\prod_{\ell \in\{1, \ldots, M\}} \gamma_{\ell}(k), \quad \forall k \in \mathbb{N}_{0} .
$$

We will assume that the $L$ channel gains $h_{\ell}(k)$ are i.i.d. and block fading with support $\Omega_{\ell}, \ell \in\{1, \ldots, L\}$. With $L$ channels fading independently of each other, state estimation accuracy can be improved by increasing the power on the channels being in a fade. However, such a simple strategy may often waste energy. A better idea is to allocate only as much power to the sensors as is necessary to attain desired performance. A lower bound would then be the allocation of power to the sensors that is sufficient to maintain stability.

We will investigate power control for two different cases: In one situation, the powers are constant; in the other case, the powers are allowed to vary with time, depending upon the current channel gains. The associated power allocation problems are presented below:

P1 While using the statistics of the channel power gains, $h_{\ell}(k)$, allocate constant power levels, $u_{\ell}(k)=u_{\ell}$, to the sensor nodes so that the sum power, $\sum_{\ell=1}^{L} u_{\ell}$, is minimized and and (8) is satisfied.

P2 Here we allow $u_{\ell}(k)$ to depend on the instantaneous channel power gains, i.e., $u_{\ell}(k)=\kappa_{\ell}(h(k))$, where $h(k) \triangleq\left(h_{1}(k), \ldots, h_{L}(k)\right)$, and the control policies $\kappa_{\ell}$ are designed using the channel gain probability density functions $p(h(k))$. The design problem addressed consists in allocating time varying power levels to the sensor nodes so that the average sum power

$$
\int_{\Omega} \sum_{\ell=1}^{L} \kappa_{\ell}(h(k)) p(h(k)) \mathrm{d} h(k), \quad \Omega \triangleq \Omega_{1} \times \cdots \times \Omega_{L}
$$

is minimized and (8) is satisfied.

Problem $\mathbf{P 2}$ is, in general, non-convex. To find (suboptimal) solutions, we will next use the generalized Karush-Kuhn-Tucker conditions, which are necessary for optimality. Expression (9) provides that

$$
\begin{aligned}
& \mathcal{P}\{s(k)=1 \mid h(k), u(k)\} \\
& =\prod_{\ell=1}^{L} \mathcal{P}\left\{\gamma_{\ell}(k)=1 \mid h_{\ell}(k), u_{\ell}(k)\right\}=\prod_{\ell=1}^{L} f_{\ell}\left(h_{\ell}(k) u_{\ell}(k)\right),
\end{aligned}
$$

so that the stability condition (8) can be expressed as

$$
\int_{\Omega}\left(\prod_{\ell=1}^{L} f_{\ell}\left(h_{\ell}(k) \kappa_{\ell}(h(k))\right)\right) p(h(k)) \mathrm{d} h(k) \geq 1-\frac{\rho}{\|A\|^{2}} .
$$

Thus, the Lagrangian of $\mathbf{P 2}$ is given by

$$
\begin{aligned}
& \mathcal{L}\left(\kappa_{1}, \kappa_{2}, \ldots, \kappa_{L}, \nu\right)=\sum_{\ell=1}^{L} \kappa_{\ell}(h) \\
& \quad-\nu\left(\prod_{\ell=1}^{L} f_{\ell}\left(h_{\ell} \kappa_{\ell}(h)\right)-\left(1-\frac{\rho}{\|A\|^{2}}\right)\right),
\end{aligned}
$$

giving the generalized Karush-Kuhn-Tucker conditions

$$
\begin{gathered}
\frac{\partial \mathcal{L}}{\partial \kappa_{\ell}}=1-\nu f_{\ell}^{\prime}\left(h_{\ell} \kappa_{\ell}(h)\right) h_{\ell} \prod_{j \neq \ell} f_{j}\left(h_{j} \kappa_{j}(h)\right)=0, \quad \nu \geq 0, \\
\int_{\Omega}\left(\prod_{\ell=1}^{L} f_{\ell}\left(h_{\ell}(k) \kappa_{\ell}(h(k))\right)\right) p(h(k)) \mathrm{d} h(k)=1-\frac{\rho}{\|A\|^{2}}
\end{gathered}
$$

where $\ell \in\{1, \ldots, L\}$. For given $\nu$ and $h(k)$, we can solve the system of $L$ equations:

$$
1-\nu f_{\ell}^{\prime}\left(h_{\ell}(k) \kappa_{\ell, \nu}(h(k))\right) h(k) \prod_{j \neq \ell} f_{j}\left(h_{j}(k) \kappa_{j, \nu}(h(k))\right)=0,
$$

numerically for $\left\{\kappa_{1, \nu}(h(k)), \ldots, \kappa_{L, \nu}(h(k))\right\}$. Amongst all possible values of $\nu$, we then find a value $\nu^{*}$ that satisfies the constraint with equality:

$\int_{\Omega}\left(\prod_{\ell=1}^{L} f_{\ell}\left(h_{\ell}(k) \kappa_{\ell, \nu^{*}}(h(k))\right)\right) p(h(k)) \mathrm{d} h(k)=1-\frac{\rho}{\|A\|^{2}}$. A sub-optimal solution to the centralized control design problem is then given by $\left\{\kappa_{1, \nu^{*}}(h), \ldots, \kappa_{L, \nu^{*}}(h)\right\}$.

\subsection{Numerical Example}

In order to illustrate properties of the power allocation strategies discussed above, we next give a simple example with Binary Phase Shift Keying (BPSK) transmission over 
$M=L$ i.i.d. block fading Rayleigh additive white Gaussian noise channels. Each sensor measurement consists of a packet containing $b$ bits. In this case, see Proakis (1995), the success probabilities can be approximated via

$$
f_{\ell}(h u)=\left(\int_{-\infty}^{\sqrt{h u}} \frac{1}{\sqrt{2 \pi}} e^{-t^{2} / 2} \mathrm{~d} t\right)^{b},
$$

with derivatives given by

$$
f_{\ell}^{\prime}(h u)=\frac{b}{2}\left(\int_{-\infty}^{\sqrt{h u}} \frac{e^{-t^{2} / 2}}{\sqrt{2 \pi}} \mathrm{d} t\right)^{b-1} \frac{e^{-h u / 2}}{\sqrt{2 \pi h u}} .
$$

Since we assume that the channels are block Rayleighfading, their power gains, $h_{\ell}(k)$, are exponentially distributed with probability density functions of the form

$$
p\left(h_{\ell}(k)\right)=\lambda_{\ell} \exp \left(-\lambda_{\ell} h_{\ell}(k)\right), \quad h_{\ell}(k) \geq 0 .
$$

It can be shown that, with this transmission model, the constant power allocation problem $\mathbf{P 1}$ is convex and the optimal power levels can be found with standard optimization software. The dynamic control law design problem, however is, in general, non-convex. For our numerical results, we use the sub-optimal policies provided by the method outlined in Section 4.1.

Fig. 2 illustrates the results for a wireless sensor estimation architecture with two channels $(L=2)$, and where $\lambda_{1}=1$ and $\lambda_{2}=2$. The figure shows a comparison of the average sum power achieved for constant and gain dependent time varying power levels as presented in Section 4.1. Clearly, if the power levels are allowed to depend upon the actual channel gains, then less sum power is needed to preserve the stability condition given in Theorem 1. Furthermore, as the number of bits per packet, $b$, and $\|A\|^{2} / \rho$ increase, the difference between the static and dynamic allocation becomes more pronounced. For example, in the situation examined it turns out that, if $\|A\|^{2} / \rho=4$ and $b=8 \mathrm{bits}$, then the constant power allocation requires almost $30 \%$ more average sum power as compared to the allocation strategy which depends upon the current channel power gain. ${ }^{2}$

\section{CONCLUSIONS}

In this work we have studied stability properties of a Kalman filter receiving sensor data over block fading wireless channels governed by power control. By the use of stochastic stability methods, we have established conditions on system parameters which ensure that the Kalman filter covariance matrix is exponentially bounded in norm. The stability condition obtained was then used as a constraint to obtain the minimum averaged sum power for the case when the powers are static and channel dependent. A simple example shows that the optimum channel gain dependent power allocation is significantly better than the allocation of static powers depending on the channel statistics only.

Future work could include studying robustness to channel estimation errors, considering more general wireless sensor network architectures, coding aspects, and correlated wireless channel models.

2 In practical situations, such a strategy will of course require estimating the channel gains; see, e.g., Ekman et al. (2002).

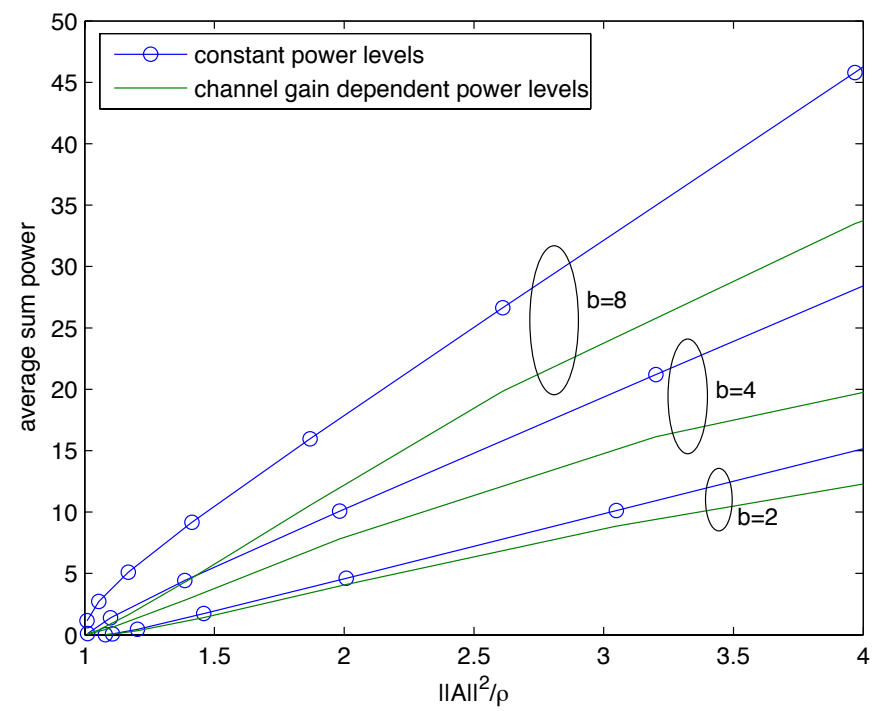

Fig. 2. Average sum power required to meet the stability condition (8) for BPSK transmission with packets of length $b=2,4$, and 8 bits.

Appendix A. PROOF OF THEOREM 1

Equation (7) provides:

$$
\mathbb{E}\{P(k+1)\}=A \mathbb{E}\{P(k)-K(k) C(k) P(k)\} A^{T}+Q, \text { (A.1) }
$$

where expectation is taken with respect to $\{\gamma\}_{0}^{k}$. Since, by assumption, $\{\gamma\}$ is white, it holds that:

$$
\begin{array}{r}
\mathbb{E}\{K(k) C(k) P(k)\}=\sum_{\{\gamma\}_{0}^{k} \in \Gamma^{k+1}} K(k) C(k) P(k) p\left(\{\gamma\}_{0}^{k}\right) \\
=\sum_{\gamma(k) \in \Gamma} \sum_{\{\gamma\}_{0}^{k-1} \in \Gamma^{k}} K(k) C(k) P(k) p\left(\{\gamma\}_{0}^{k-1}\right) p(\gamma(k)),
\end{array}
$$

where $\Gamma=\{0,1\}^{L}$ and $\Gamma^{k}=\Gamma \times \cdots \times \Gamma$. If we now condition upon $s(k)$, which depends upon $\gamma(k)$ but is independent of $\{\gamma\}_{0}^{k-1}$, then we obtain

$$
\begin{aligned}
\mathbb{E}\{K(k) C(k) P(k)\} \\
=\mathbb{E}\{K(k) C(k) P(k) \mid s(k)=1\} \mathcal{P}\{s(k)=1\} \\
\quad+\mathbb{E}\{K(k) C(k) P(k) \mid s(k)=0\}(1-\mathcal{P}\{s(k)=1\}),
\end{aligned}
$$

where the (conditional) expectations are taken with respect to the random variables $\{\gamma\}_{0}^{k-1}$.

For $s(k)=1$, we can proceed as in the proof of the corollary in Katayama (1976) and bound:

$$
\begin{aligned}
& K(k) C(k) P(k) \\
& \quad=P(k) C(k)^{T}\left(C(k) P(k) C(k)^{T}+R\right)^{-1} C(k) P(k) \\
& =P(k)-\left(P(k)^{-1}+C(k)^{T} R^{-1} C(k)\right)^{-1} \succeq P(k)-\delta I,
\end{aligned}
$$

where $\delta^{-1}=\min \operatorname{eigs}\left(C(k)^{T} R^{-1} C(k)\right)>0$, since in this case it holds that $C(k)^{T} R^{-1} C(k) \succ 0$.

On the other hand, for the events where $s(k)=0$, we have:

$$
\begin{aligned}
& K(k) C(k) P(k) \\
& =P(k) C(k)^{T}\left(C(k) P(k) C(k)^{T}+R\right)^{-1} C(k) P(k) \succeq 0 .
\end{aligned}
$$


Substitution of (A.3) and (A.4) into (A.2) provides that: $\mathbb{E}\{K(k) C(k) P(k)\} \succeq(\mathbb{E}\{P(k)\}-\delta I) \mathcal{P}\{s(k)=1\}$

so that, by (A.1), we obtain:

$$
\begin{aligned}
\mathbb{E}\{P(k+1)\} \preceq(1-\mathcal{P}\{s(k)=1\}) A \mathbb{E}\{P(k)\} A^{T} \\
+Q+\mathcal{P}\{s(k)=1\} \delta A A^{T} \\
\preceq(1-\mathcal{P}\{s(k)=1\}) A \mathbb{E}\{P(k)\} A^{T} \\
+Q+\delta A A^{T} .
\end{aligned}
$$

If we now introduce $V_{k} \triangleq \operatorname{tr} P(k)$, which is non-negative since $P(k) \succeq 0$, then (A.5) gives:

$$
\begin{aligned}
\mathbb{E}\left\{V_{k+1}\right\} & \leq(1-\mathcal{P}\{s(k)=1\}) \operatorname{tr}\left(A \mathbb{E}\{P(k)\} A^{T}\right)+\beta^{\prime} \\
& \leq(1-\mathcal{P}\{s(k)=1\})\|A\|^{2} \operatorname{tr} \mathbb{E}\{P(k)\}+\beta^{\prime} \\
& \leq \rho \mathbb{E}\left\{V_{k}\right\}+\beta^{\prime},
\end{aligned}
$$

where we have used Corollary 8.4.10 and Fact 8.12.29 in Bernstein (2009), the condition (8), and where

This gives:

$$
\beta^{\prime} \triangleq \operatorname{tr}\left(Q+\delta A A^{T}\right) \in[0, \infty) .
$$

$$
\mathbb{E}\left\{V_{k}\right\} \leq \rho^{k} \operatorname{tr} P_{0}+\beta^{\prime} \sum_{i=0}^{k-1} \rho^{i}
$$

Since $P(k)$ is a covariance matrix and, hence, $P(k) \succeq 0$, we have

and (A.6) gives:

$$
V_{k}=\operatorname{tr} P(k) \geq\|P(k)\|
$$

$$
\mathbb{E}\{\|P(k)\|\} \leq \rho^{k} \operatorname{tr} P_{0}+\beta^{\prime} \frac{1-\rho^{k}}{1-\rho} .
$$

The result follows from the bound $0<1-\rho^{k} \leq 1$.

\section{REFERENCES}

Anderson, B.D.O. and Moore, J. (1979). Optimal Filtering. Prentice Hall, Englewood Cliffs, NJ.

Bernstein, D.S. (2009). Matrix Mathematics. Princeton University Press, Princeton, N.J., 2nd edition.

Björnemo, E. (2009). Energy Constrained Wireless Sensor Networks: Communication principles and sensing aspects. Ph.D. thesis, Uppsala University, http://www.signal.uu.se/Publications/ptheses.html, Department of Engineering Sciences, Signals and Systems, P.O. Box 534, SE751 21 UPPSALA.

Björnemo, E., Ahlén, A., and Johansson, M. (2007). On the energy-efficiency of cooperative MIMO in Nakagami fading wireless sensor networks. In Proc. 41st Asilomar Conf. Signals, Systems, and Computers. Monterey, CA.

Dey, S., Leong, A.S., and Evans, J.S. (2009). Kalman filtering with faded measurements. Automatica, 45, 2223-2233.

Ekman, T., Sternad, M., and Ahlén, A. (2002). Unbiased power prediction of Rayleigh fading channels. In Proc. Vehicular Technology Conf., volume 1, 280-284.

Gharavi, H. and Kumar, S.P. (2003). Special section on sensor networks and applications. Proc. IEEE, 91(8), 1151-1152.

Goldsmith, A. (2005). Wireless Communications. Cambridge University Press.

Gupta, V., Martins, N.C., and Baras, J.S. (2009). Optimal output feedback control using two remote sensors over erasure channels. IEEE Trans. Automat. Contr., 54(7), 1463-1476.
Havinga, P., Hou, J.C., and Zhao, F. (2004). Wireless sensor networks [guest ed.]. IEEE Wireless Commun., 11(12), 4-5.

Hespanha, J.P., Naghshtabrizi, P., and Xu, Y. (2007). A survey of recent results in networked control systems. Proc. IEEE, 1(95), 138-162.

Ilyas, M., Mahgoub, I., and Kelly, L. (2004). Handbook of Sensor Networks: Compact Wireless and Wired Sensing Systems. CRC-Press, Inc, Boca Raton, FL, USA.

Johansson, M., Björnemo, E., and Ahlén, A. (2007). Fixed link margins outperform power control in energylimited wireless sensor networks. In Proc. IEEE Int. Conf. Acoust. Speech Signal Process., volume 3, 513-516. Honolulu, HI.

Katayama, T. (1976). On the matrix Riccati equation for linear systems with a random gain. IEEE Trans. Automat. Contr., 21(5), 770-771.

Kluge, S., Reif, K., and Brokate, M. (2010). Stochastic stability of the extended Kalman filter with intermittent observations. IEEE Trans. Automat. Contr., 55(2), 514518.

Luenberger, D.G. (1969). Optimization by Vector Space Methods. Wiley-Interscience, New York, N.Y.

Nair, G.N., Fagnani, F., Zampieri, S., and Evans, R.J. (2007). Feedback control under data rate constraints: An overview. Proc. IEEE, 95(1), 108-137.

Proakis, J.G. (1995). Digital Communications. McGrawHill, New York, N.Y., 3rd edition.

Quevedo, D.E., Ahlén, A., and Østergaard, J. (2010). Energy efficient state estimation with wireless sensors through the use of predictive power control and coding. IEEE Trans. Signal Processing, 58(9), 4811-4823.

Rohr, E., Marelli, D., and Fu, M. (2010). Statistical properties of the error covariance in a Kalman filter with random measurement losses. In Proc. IEEE Conf. Decis. Contr. Atlanta, GA USA.

Schenato, L. (2008). Optimal estimation in networked control systems subject to random delay and packet drop. IEEE Trans. Automat. Contr., 53(5), 1311-1317.

Schenato, L., Sinopoli, B., Franceschetti, M., Poolla, K., and Sastry, S.S. (2007). Foundations of control and estimation over lossy networks. Proc. IEEE, 95(1), 163187.

Shen, X., Zhang, Q., and Caiming Qiu, R. (2007). Wireless sensor networking [guest ed.]. IEEE Wireless Commun., 14(6), 4-5.

Shi, L., Epstein, M., and Murray, R.M. (2010). Kalman filtering over a packet-dropping network: A probabilistic perspective. IEEE Trans. Automat. Contr., 55(3), 594604.

Sinopoli, B., Schenato, L., Franceschetti, M., Poolla, K., Jordan, M.I., and Sastry, S.S. (2004). Kalman filtering with intermittent observations. IEEE Trans. Automat. Contr., 49(9), 1453-1464.

Wen, R., Mareels, I., and Krongold, B. (2008). Optimal power analysis for network lifetime balance in hierarchy networks. In Proc. IFAC World Congr. Seoul, Korea.

Xiao, J.J., Cui, S., Luo, Z.Q., and Goldsmith, A.J. (2008). Linear coherent decentralized estimation. IEEE Trans. Signal Processing, 56(2), 757-770.

Xie, L. and Xie, L. (2008). Stability of a random Riccati equation with Markovian binary switching. IEEE Trans. Automat. Contr., 53(7), 1759-1764. 